\title{
Quantum Gravity Effects in Statistical Mechanics with Modified Dispersion Relation
}

\author{
Shovon Biswas ${ }^{1}$ and Mir Mehedi Faruk (D) $^{2,3}$ \\ ${ }^{1}$ Department of Electrical and Electronic Engineering, Bangladesh University of Engineering and Technology, Dhaka 1000, Bangladesh \\ ${ }^{2}$ Bose Centre for Advanced Study and Research in Natural Science, University of Dhaka, Dhaka, Bangladesh \\ ${ }^{3}$ Physics Department, McGill University, Montreal, QC, Canada H3A 2T8
}

Correspondence should be addressed to Mir Mehedi Faruk; muturza3.1416@gmail.com

Received 25 September 2017; Revised 14 December 2017; Accepted 15 February 2018; Published 18 March 2018

Academic Editor: Elias C. Vagenas

Copyright (c) 2018 Shovon Biswas and Mir Mehedi Faruk. This is an open access article distributed under the Creative Commons Attribution License, which permits unrestricted use, distribution, and reproduction in any medium, provided the original work is properly cited. The publication of this article was funded by SCOAP ${ }^{3}$.

Planck scale inspired theories which are also often accompanied with maximum energy and/or momentum scale predict deformed dispersion relations compared to ordinary special relativity and quantum mechanics. In this paper, we resort to the methods of statistical mechanics in order to determine the effects of a deformed dispersion relation along with an upper bound in the partition function that maximum energy and/or momentum scale can have on the thermodynamics of photon gas. We also analyzed two distinct quantum gravity models in this paper.

\section{Introduction}

Different quantum gravity approaches such as string theory, loop quantum gravity, noncommutative geometry, doubly special relativity (DSR), and generalized uncertainty principle (GUP) suggest the existence of a minimal length scale of the order of Planck length, $l_{p}=\sqrt{G \hbar / c^{3}}$. This length and its inverse, the Planck energy $E_{p}$, mark thresholds beyond which the old description of space-time breaks down and qualitatively new phenomena are expected to appear [1]. DSR is a very well-known approach to deal with minimal length situations. DSR type theories predict a modified dispersion relation compared to usual dispersion relation of special relativity. But one should notice that DSR can be formulated in different approaches. One of the approaches [2] which maintain a varying speed of light (VSL) scenario can be entitled with maximum momenta or maximum energy depending on the value of the parameter which describes the minimal length scale of the theory (we will refer to this as model A in this manuscript). Another possible DSR scenario $[3,4]$ in which the speed of light is constant is also heavily studied with the help of nonlinear realizations of the Lorentz group (this is referred to as model 1 in the rest of the paper). A point to note, these different models predict different dispersion relations and distinct physical phenomena. The implication of nonzero minimal length has been considered recently in different contexts that include harmonic oscillators [5-10], hydrogen atoms [11-14], gravitational quantum wells [15], the Casimir effect [16, 17], particles scattering $[18,19]$, relativistic thermodynamics of ideal fluids [20], blackbody radiation [21], and Unruh effect $[22,23]$. It has been reported [24] and well established that a consistent marriage of ideas of quantum mechanics and gravitation needs a noncommutative description of spacetime to avoid the paradoxical condition of creating a black hole for an event that is sufficiently localized in space-time. As DSR admits noncommutative space-time, DSR theory fits the criterion for being a quantum gravity framework.

Invariant energy scales, that is, Planck scale modified dispersion relations, are usually identified with maximum energy or maximum momenta or both $[1,2,25]$. It is interesting to note that dispersion modifications can lead to many further predictions which can be tested in a series of experiments such as gamma ray burst observation [26] or inflationary cosmology [27]. In this spirit, lots of studies are done on statistical thermodynamics of quantum gases $[3,4,28,29]$. 
One has to be very careful while calculating the partition function of the quantum gases within these models as different models change the structure of the partition function. For instance, in the case of a massless boson, model A changes both the integration measure and the upper limit of the partition function [2], while in model 1 only the upper limit is changed [3, 4, 30]. Different errors have been noticed in several manuscripts while evaluating the partition function for quantum gases. For example, in [3, 28], Maxwell-Boltzmann distribution functions are used to calculate the thermodynamics of photon gas. Reference [3] is based on model 1. One of us (MMF) has recently made an attempt [4] to evaluate the partition function of photon gas with a correct distribution function, that is, Bose-Einstein distribution function just as in [2]. But while calculating the partition function in a previous manuscript [4], an error has been noticed, which will be corrected in this paper (we have adopted $\hbar=c=k_{B}=1$ ). The deformed dispersion relation predicted by model 1 can be written as $[1,3]$ (we have adopted $\hbar=c=k_{B}=1$ )

$$
E^{2}=p^{2}+m^{2}\left(1-\frac{E}{E_{p}}\right)^{2} .
$$

Here, $E$ and $p$ are the energy and the magnitude of the three momenta of the particle, respectively, while $m$ is the mass of the particle and $E_{p}$ is the Planck energy. Now, for massless particles, the dispersion reduces to $E=p$. That is why an upper energy bound in energy is equivalent to an upper energy bound in momentum in this case. However, in model A, the dispersion relation for massless particles [2] takes the form

$$
p=\frac{E}{1+\lambda E}
$$

where $|\lambda| \propto 1 / E_{P}$, so $\lambda$ can be both positive and negative. Now, when $\lambda>0,(2)$ implies a maximum momentum $p_{\max }=$ $\lambda^{-1}$, which remains invariant under deformed transformation laws. In contrast, $\lambda<0$ corresponds to an energy upper bound for photons. So, in case of model A, we find an upper bound either in momentum or in energy, whereas in model 1 we see an upper bound both in energy and in momentum. In this manuscript, we will solve the partition function for photon gas with model 1 .

Now, another well studied modified dispersion relation based on a phenomenological study is $[30,31]$

$$
E^{2}=p^{2}\left[1-\alpha\left(\frac{E}{E_{P}}\right)^{n}\right]+m^{2} .
$$

Here, $E_{P}$ is the Planck energy and $\alpha$ is a coefficient of order 1 , whose precise value depends upon the considered quantum gravity model, while $n$, the lowest power in Planck's length leading to a nonvanishing contribution, is also a model-dependent quantity. This type of dispersion relation is observed in loop quantum gravity [31]. In [31-34], it has been argued that $n$ can be chosen as $n=1$ or $n=2$. See [31] to understand the different physical scenarios with $n=1$ and $n=2$. Now, choosing $m=0$ and following the spirit of
Zhang et al. [2], we find out from (3) that $E_{P}>0$ indicates an upper bound in energy $E_{\max }=E_{P}$. A bound in momentum can be obtained with $E_{P}<0$, which is unphysical, so we discard the scenario. We must note that Camacho and Macías [30] have already visited the thermodynamics of photon gas within this model. But they did not take into account the upper bound of maximum energy while calculating the partition function. Any model aimed at describing the behavior of thermodynamic quantities near Planck scale must take into account the effect of the upper bound. In this paper, we will also visit the thermodynamics of photon gas with this deformed dispersion relation, but with maximum energy bound as argued above. We will refer to this model as model 2 in this paper. We have found that, due to this maximum energy bound, the final results of this model are drastically changed compared with what was reported before. A remarkable similarity is noticed between the results of this Lorentz violating model and another recently reported Lorentz violating model [35].

\section{Thermodynamics of Photon Gas with Deformed Dispersion Relation in Model 1}

2.1. Partition Function. The expression of the partition function in usual statistical mechanics for massless bosons in grand canonical ensemble can be obtained [36]:

$$
\ln Z=-\sum_{i} \ln \left(1-e^{-\beta E_{i}}\right)
$$

Here, $\beta=1 / T$. Now, changing the sum to integral, we find out

$$
\ln Z=-\frac{1}{(2 \pi)^{3}} \iint d^{3} r d^{3} p \ln \left(1-e^{-\beta E}\right)
$$

In [1], Magueijo and Smolin proposed a DSR model where the dispersion relation for a massive particle takes the form

$$
E^{2}=p^{2}+m^{2}\left(1-\frac{E}{E_{p}}\right)^{2} .
$$

For massless photon gas, (6) takes the usual form $E=p$. Take a look at [3] to see how the phase space volume remains unaltered in this model. One might also wonder whether phase space is invariant under canonical transformation. Canonical transformation are those which keep the form of Hamiltonian's equation invariant. In case of usual special relativity (SR), the Hamiltonian equation for massless particles is $E=p$. Now, of course, any canonical transformation must keep the form of this equation invariant. And it is known that these canonical transformations also keep the usual phase space volume $\left(\int d x d p\right)$ invariant. In case of model 1 , the dispersion relation changes due to the existence of the Planck scale [see (1)]. Now, if we only consider the massless case of model 1 , one can see then that the dispersion relation is nothing but (1) (just like the usual SR scenario). Therefore, in the massless scenario, the dispersion relation of model 
1 reduces to the usual special relativity. As the dispersion relation (Hamiltonian's equation) is of the usual form (same as special relativity), the canonical transformations associated with the massless case of model 1 are the same as the usual special relativity scenarios. And we already know that these canonical transformations not only keep the Hamiltonian equation invariant, but also keep the phase space volume $\left(\int d x d p\right)$ invariant. Therefore, in this case, it is enough to take the usual phase space volume as phase space volume for the massless case of model 1 . But a point to note is that it is only true for the massless case. In case of massive particles, the dispersion relation (Hamiltonian equation) changes [see (1)] and therefore the canonical transformation changes compared to the usual SR. As a result, the phase space volume also changes. But as we are dealing only with the massless scenarios, the phase space volume mentioned in here is justified. Now, due to the presence of an upper bound in energy, $E_{P}$ in this model, the partition function becomes

$$
\ln Z=-\frac{4 \pi V}{(2 \pi)^{3}} \int_{0}^{E_{P}} E^{2} \ln \left(1-e^{-\beta E}\right) d E .
$$

Here, $V$ is the total volume of the system. Equation (7) can be written as

$$
\ln Z=\frac{4 \pi V}{(2 \pi)^{3}} \frac{T^{3}}{3} f_{4},
$$

where the function $f_{n}$ is defined in the Appendix. (In order to calculate (7), one can choose $x=\beta E$. As a result, the upper limit of integration should also be changed accordingly. Although a correct distribution function, i.e., Bose-Einstein distribution, was taken instead of Maxwell-Boltzmann distribution in our study [4], the change of the upper limit of integration with respect to this variable shift was not mistakenly taken into account. Once this variable shift is also taken into account in the upper bound of the integration, one finds out that the upper bound of the integration contains a temperature-dependent term, which was completely absent in [4].)

2.2. Thermodynamic Quantities. Thermodynamic quantities can be derived easily from the partition function following the simple and effective rules of statistical mechanics [36]. For example, the internal energy is related to the partition function by

$$
U=T^{2}\left(\frac{\partial \ln Z}{\partial T}\right)_{V}=\frac{4 \pi V}{(2 \pi)^{3}} T^{4}\left[f_{4}+\frac{T}{3} f_{4}^{\prime}\right] .
$$

The pressure can be found from the relation

$$
P V=T \ln Z=\frac{4 \pi}{(2 \pi)^{3}} \frac{T^{4}}{3} f_{4} .
$$

We see that the well-known relationship between pressure and energy $P=U / 3 \mathrm{~V}$ is not valid anymore as obtained in the usual statistical mechanics. Another important thermodynamic quantity is entropy $S$ :

$$
S=-\left(\frac{\partial F}{\partial T}\right)_{V}=\frac{4 \pi V}{(2 \pi)^{3}} T^{3}\left[\frac{4}{3} f_{4}+\frac{T}{3} f_{4}^{\prime}\right],
$$

where $F=-T \ln Z$. As $T \rightarrow 0$, we notice $S \rightarrow 0$ indicating that Nernst's postulate is valid. Finally, specific heat can be obtained from the relation

$$
C_{V}=\left(\frac{\partial U}{\partial T}\right)_{V}=\frac{4 \pi V}{(2 \pi)^{3}} T^{4}\left[\frac{4 f_{4}}{T}+\frac{8}{3} f_{4}^{\prime}+\frac{T}{3} f_{4}^{\prime \prime}\right] .
$$

All the thermodynamic quantities reduce to the usual results of statistical mechanics in the limit $E_{P} \rightarrow \infty$ [36].

\section{Thermodynamics of Photon Gas with Deformed Dispersion Relation in Model 2}

3.1. The Partition Function. Now, let us focus on the model based on the phenomenological study, first discussed in detail by Amelino-Camelia [26, 31]. Later, thermodynamics of photon gas were studied within this model by Camacho and Marcias [30]. As discussed in the Introduction, Camacho and Marcias did not take into account the upper energy bound that exists in this model, while evaluating the partition function. In this section, we evaluate the partition function in the presence of the upper bound.

The modified dispersion relation of massless bosons in this model is

$$
E^{2}=p^{2}\left[1-\alpha\left(\frac{E}{E_{P}}\right)^{n}\right] \Longrightarrow \frac{1}{p^{2}}=\frac{1-\alpha\left(E / E_{P}\right)^{n}}{E^{2}}
$$

Now, it is clear from the above equation with $p \rightarrow \infty$ that $E$ tends to $\left(1 / \alpha^{1 / n}\right) E_{P}$, that is, the maximum value of $E$, considering $E_{P}>0$. As $\alpha \sim 1[26,31]$, we find out $E_{\max }=E_{P}$.

The partition function can be equivalently written as

$$
\ln Z=-\int d E \rho(E) \ln \left(1-e^{-\beta E}\right)
$$

where $\rho(E)$ is the density of states [30]:

$$
\rho(E)=\frac{4 \pi V}{(2 \pi)^{3}} E^{2}\left[1+\left(n+\frac{3}{2}\right)\left(\frac{E}{E_{p}}\right)^{n}\right] .
$$

Based on the above observation, we can write the partition function:

$$
\begin{aligned}
& \ln Z=-\frac{4 \pi V}{(2 \pi)^{3}} \\
& \cdot \int_{0}^{E_{p}} E^{2}\left[1+\left(n+\frac{3}{2}\right)\left(\frac{E}{E_{p}}\right)^{n}\right] \ln \left[1-e^{-E / T}\right] d E .
\end{aligned}
$$

Equation (16) can be rewritten as

$$
\begin{aligned}
\ln Z & =\frac{4 \pi V}{(2 \pi)^{3}}\left[I_{4}+\frac{(n+3 / 2)}{E_{p}^{n}} I_{n+4}\right] \\
& =\frac{4 \pi V T^{3}}{(2 \pi)^{3}}\left[\frac{f_{4}}{3}+\frac{(n+3 / 2)}{n+3}\left(\frac{T}{T_{p}}\right)^{n} f_{n+4}\right] .
\end{aligned}
$$


3.2. Thermodynamic Quantities. Just like in Section 2.2, the thermodynamic quantities can be derived from the partition function.

Internal Energy

$$
\begin{aligned}
U & =T^{2}\left(\frac{\partial \ln Z}{\partial T}\right)_{V}=\frac{4 \pi V}{(2 \pi)^{3}} T^{4}\left[f_{4}+\frac{T}{3} f_{4}^{\prime}\right. \\
& \left.+\left(n+\frac{3}{2}\right)\left(\frac{T}{T_{p}}\right)^{n}\left\{f_{n+4}+T \frac{f_{n+4}^{\prime}}{n+3}\right\}\right] .
\end{aligned}
$$

Pressure

$$
P=\frac{4 \pi T^{3}}{(2 \pi)^{3}}\left[\frac{f_{4}}{3}+\frac{(n+3 / 2)}{n+3}\left(\frac{T}{T_{p}}\right)^{n} f_{n+4}\right] .
$$

Entropy

$$
\begin{aligned}
S= & \frac{4 \pi V}{(2 \pi)^{3}} T^{3}\left[\frac{4}{3} f_{4}+\frac{T}{3} f_{4}^{\prime}\right. \\
& \left.+\frac{(n+3 / 2)}{n+3}\left(\frac{T}{T_{p}}\right)^{n}\left\{(n+4) f_{n+4}+T f_{n+4}^{\prime}\right\}\right] .
\end{aligned}
$$

Specific Heat

$$
\begin{aligned}
C_{V} & =\left(\frac{\partial U}{\partial T}\right)_{V}=\frac{4 \pi V}{(2 \pi)^{3}} T^{4}\left[\frac{4 f_{4}}{T}+\frac{8}{3} f_{4}^{\prime}+\frac{T}{3} f_{4}^{\prime \prime}\right. \\
& +\left(n+\frac{3}{2}\right)\left(\frac{T}{T_{p}}\right)^{n} \\
& \left..\left\{\frac{(n+4) f_{n+4}}{T}+\frac{2(n+4)}{n+3} f_{n+4}^{\prime}+\frac{T}{n+3} f_{n+4}^{\prime \prime}\right\}\right] .
\end{aligned}
$$

The thermodynamic quantities of this model also coincide with the results of the usual statistical mechanics in the limit $E_{P} \rightarrow \infty$. The presence of an upper bound in the partition function drastically changes the final outcome of this model. Several other observations will be noted in the next section.

\section{Discussion and Conclusion}

We are now in a position to present the results, obtained in the previous two sections. The results have been obtained using Mathematica.

In Figure 1, we have plotted the pressure obtained from model 1 and model 2, with $n=1$ and $n=2$, and performed a comparison with the usual results of special relativity (SR). In the study of model 1 (DSR), we have noticed that the values of pressure exactly coincide with SR results in low temperature regions but show deviation from the usual result at a high temperature limit. At any given temperature, the thermodynamic quantities in the DSR model pick smaller values compared to the usual result in that temperature. This is expected in the DSR model, as the dispersion relation is unchanged compared to SR, and due to the presence of an upper energy scale, the total number of microstates becomes less than SR. Although this result has been reported before in [4], we should note that, due to the correction (we have adopted $\hbar=c=k_{B}=1$ ) we have presented in the current paper in evaluating the partition function for the DSR model, the resulting thermodynamic quantities take lesser values compared to the previous study [4].

In the previous study with model 2, Camacho and Marcias have predicted that pressure and other thermodynamic quantities are always greater than the SR results. This scenario is drastically changed in our study as we have taken into account the existence of an upper energy bound $E_{P}$. At low temperature, the predicted pressure of this model coincides with the usual result as it should be, but after a certain temperature, it starts taking a larger value compared to the usual results. Near $T=0.5 T_{P}$, model 2 result intersects with $S R$ result and afterwards the predicted outcome takes a lesser value compared to the usual result of SR. This is due to the cut-off that we considered in our calculation. Such property is also noticed in a recent Lorentz violating study [35] with an upper cut-off. This characteristic is also noticed in case of other thermodynamic quantities of model 2 (see Figures 1(b) and $1(\mathrm{c})$ ). This type of unique characteristic is not noticed when an upper bound in the partition function [30] is not present.

The different thermodynamic quantities for ideal photon gas in SR theory maintain specific relations, such as pressureenergy relation $((P V=3 U))$, internal energy-free energy relation $(U=-3 F)$, and specific heat-entropy relation $\left(3 S=C_{v}\right)$ [36]. None of them remains valid for any of these models with an upper energy bound. In the previous study with model 1 [4], it was reported that such relations are maintained, but this was due to the error we pointed out previously (i.e., we have adopted $\hbar=c=k_{B}=1$ ). Once we have taken care of the error, the relations are not maintained anymore.

In this paper, we have studied the thermodynamics of photon gas with two different models: model 1 is based on DSR that preserves Lorentz algebra and model 2 is based on a phenomenological Lorentz violating quantum gravity model. But due to the presence of the deformed density of states and an upper bound, the quest of solving the partition function becomes complicated. The results of our calculation from both models coincide with the known results [36] of SR theory in the limit $E_{P} \rightarrow \infty$, as expected. In model 1, we have seen that the growth of all the thermodynamic quantities is slower compared to SR. This is due to the presence of an invariant energy upper bound in this theory. Therefore, the microstates can attain energies only up to a finite cutoff, whereas in the SR theory, the microstates can attain energies up to infinity. The current study provides significant improvement of the previous study based on model 1 . We have not only corrected the mathematical error of the previous study [4] but also noticed that the final outcome is also changed compared to [4] due to the correction. The complete evaluation of (19), (20), and (21) reveals in Figure 1 that the thermodynamic quantities in this model change more slowly with temperature than previously reported. Regarding the second model under consideration, it was reported by 


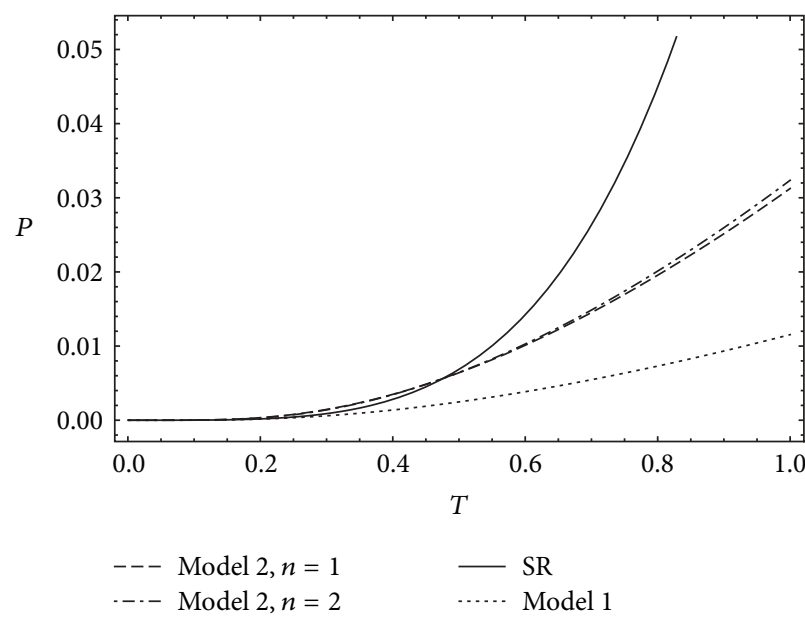

(a) Pressure $P$ versus temperature

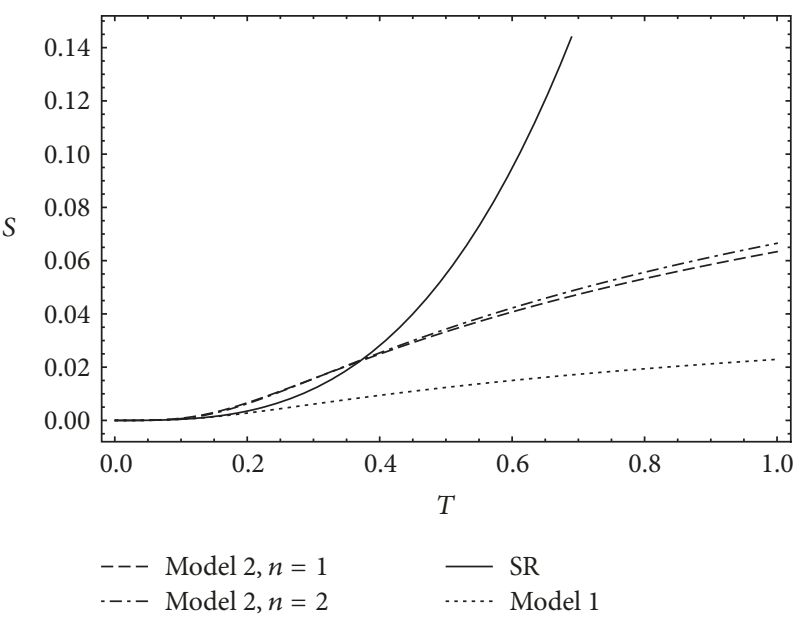

(b) Entropy $S$ versus temperature

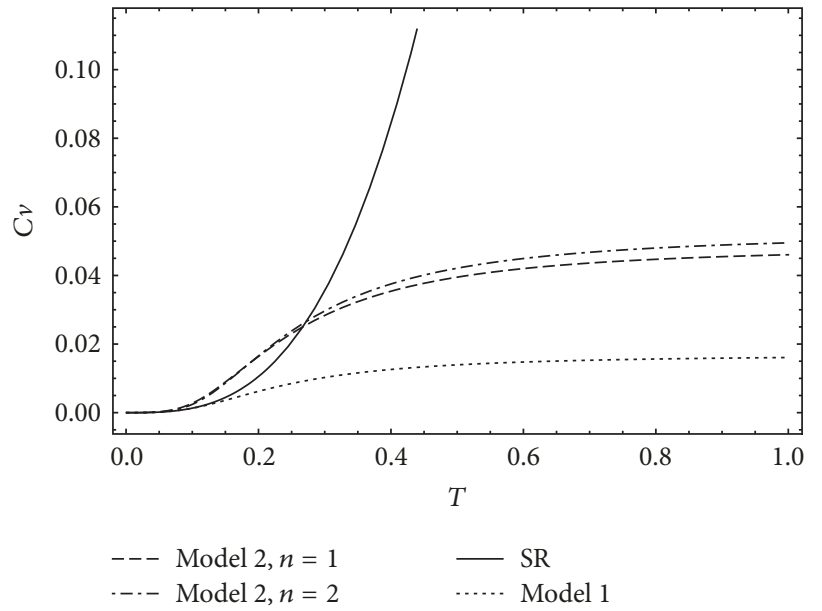

(c) Specific heat $C_{v}$ versus temperature

FIGURE 1: In this figure, we have plotted pressure $P$, entropy $S$, and specific heat $C_{v}$ of photon gas against temperature $T$ for model 1 (DSR) and model 2 with $n=1$ and $n=2$. The results of models 1 and 2 have been obtained numerically using Mathematica. We also used the Planck units, and the corresponding parameters take the following values: $E_{P}=1, k_{B}=1, V=1, h=1$, and $c=1$. In this scale, $T=1$ is the Planck temperature, $T_{P}$.

Camacho and Macías that the thermodynamic quantities of photon gas would have a higher value in contrast to special relativity in this model. But as we have introduced an upper bound in this model, we have found the result to be quite the opposite. Though the thermodynamic quantities have greater values than those compared to SR initially, at a certain temperature, both graphs intersect and afterwards the thermodynamic quantities in this Lorentz violating model attain a lower value compared to SR. This exact behavior was also noticed in a recent study based on another Lorentz violating model [35]. So, these can be taken as signatures for future experiments to detect space-time behavior near Planck scale, whether it admits Lorentz violating or preserving structure. This property was not noticed in the previous study based on model 2 due to the absence of an upper energy bound. Therefore, a significant improvement of statistical mechanics study is achieved based on model 2 .
As we have seen that the deformed dispersion relation and upper energy bound cause the modification of the thermodynamical behavior as well as the equation of state of the massless bosons such as photon gas, we are most certain it will also change such characteristics for massive bosons. We are planning to reexamine the models for massive bosons to find out the properties of Bose-Einstein condensates near Planck scale. This may have an interesting effect in the scalar field dark matter model [37], where the dark matter particle is conjectured as a spin- 0 boson. In a quite similar spirit, we may study the case of fermions in these models with upper energy models. This will yield an intriguing situation in astrophysics, since the Chandrasekhar mass-radius relation for white dwarfs is a direct consequence of the Fermi statistics. We are in the process of generalizing standard cosmology results within these models. 


\section{Appendix}

\section{Evaluation of Integrals}

The integrals that appear in this paper are of the form

$$
I_{n}=-\int_{0}^{E_{p}} E^{n-2} \ln \left(1-e^{-E / k_{B} T}\right) d E .
$$

Note that we have put back Boltzmann constant $k_{B}$. By integrating by parts, we have

$$
I_{n}=-\frac{E_{p}^{n-1}}{n-1} \ln \left(1-e^{-T_{p} / T}\right)+\frac{\left(k_{B} T\right)^{-1}}{n-1} I,
$$

where

$$
\begin{aligned}
I & =\int_{0}^{E_{p}} \frac{E^{n-1} e^{-E / k_{B} T}}{1-e^{-E / k_{B} T}} d E \\
& =\int_{0}^{E_{p}}\left(\sum_{m=1}^{\infty} E^{n-1} e^{-m E / k_{B} T} d E\right)
\end{aligned}
$$

and $T_{p}=E_{p} / k_{B}$ is the Planck temperature. In the scale $k_{B}=1$ which has been used throughout the paper, we have $E_{p}=T_{p}$. The first term tends to zero because of the very large value of $E_{p}$. Substituting $t=m E / T$ and finally changing the order of sum and integral, we obtain

$$
I=(T)^{n} \sum_{m=1}^{\infty} \int_{0}^{m T_{p} / T} \frac{t^{n-1} e^{-t}}{m^{n}} d t .
$$

Therefore,

$$
I=(T)^{n} \sum_{m=1}^{\infty} \frac{\gamma\left(n, m T_{p} / T\right)}{m^{n}},
$$

where $\gamma(a, z)$ is the lower incomplete gamma function which is related to the gamma function by $\gamma(a, z)=\Gamma(a)-\Gamma(a, z)$, where $\Gamma(a, z)$ is the upper incomplete gamma function defined by $\int_{z}^{\infty} x^{a-1} e^{-x} d x$. Finally, we have

$$
I_{n}=\frac{(T)^{n-1}}{n-1} f_{n}
$$

where

$$
f_{n}=\sum_{m=1}^{\infty} \frac{\gamma\left(n, m T_{p} / T\right)}{m^{n}} .
$$

In the limit $E_{p} \rightarrow \infty, f_{n}$ becomes $f_{n}=\zeta(n) \Gamma(n)$. Finally, let us note the derivative of $f_{n}$ with respect to $T$ :

$$
f_{n}^{\prime}=-\frac{T_{p}^{n}}{T^{n+1}\left(\exp \left(T_{p} / T\right)-1\right)} .
$$

Note that both at $T \rightarrow 0$ and at $T_{p} \rightarrow \infty, f^{\prime} \rightarrow 0$.

\section{Conflicts of Interest}

The authors declare that there are no conflicts of interest regarding the publication of this paper.

\section{Acknowledgments}

Shovon Biswas would like to thank Supantho Raxit for proofreading the manuscript.

\section{References}

[1] J. Magueijo and L. Smolin, "Lorentz invariance with an invariant energy scale," Physical Review Letters, vol. 88, Article ID 190403, 2002.

[2] X. Zhang, L. Shao, and B.-Q. Ma, "Photon gas thermodynamics in doubly special relativity," Astroparticle Physics, vol. 34, pp. 840-845, 2011.

[3] S. Das and D. Roychowdhury, "Thermodynamics of a photon gas with an invariant energy scale," Physical Review D: Particles, Fields, Gravitation and Cosmology, vol. 81, no. 8, 2010.

[4] M. M. Faruk and M. M. Rahman, "Planck scale effects on the thermodynamics of photon gases," Physical Review D: Particles, Fields, Gravitation and Cosmology, vol. 94, no. 10, 2016.

[5] A. Kempf, G. Mangano, and R. B. Mann, "Hilbert space representation of the minimal length uncertainty relation," Physical Review D: Particles, Fields, Gravitation and Cosmology, vol. 52, no. 2, pp. 1108-1118, 1995.

[6] A. Kempf, "Non-pointlike particles in harmonic oscillators," Journal of Physics A: Mathematical and General, vol. 30, no. 6, pp. 2093-2101, 1997.

[7] L. N. Chang, D. Minic, N. Okamura, and T. Takeuchi, "Exact solution of the harmonic oscillator in arbitrary dimensions with minimal length uncertainty relations," Physical Review D: Particles, Fields, Gravitation and Cosmology, vol. 65, Article ID 125027, 2002.

[8] C. Quesne and V. M. Tkachuk, "Harmonic oscillator with nonzero minimal uncertainties in both position and momentum in a SUSYQM framework," Journal of Physics A: Mathematical and General, vol. 36, no. 41, pp. 10373-10389, 2003.

[9] C. Quesne and V. M. Tkachuk, "More on a SUSYQM approach to the harmonic oscillator with nonzero minimal uncertainties in position and/or momentum," Journal of Physics A: Mathematical and General, vol. 37, no. 43, pp. 10095-10113, 2004.

[10] C. Quesne and V. M. Tkachuk, "Dirac oscillator with nonzero minimal uncertainty in position," Journal of Physics A: Mathematical and General, vol. 38, no. 8, pp. 1747-1765, 2005.

[11] F. Brau, "Minimal length uncertainty relation and the hydrogen atom," Journal of Physics A: Mathematical and General, vol. 32, no. 44, pp. 7691-7696, 1999.

[12] S. Benczik, L. N. Chang, D. Minic, and T. Takeuchi, "Hydrogenatom spectrum under a minimal-length hypothesis," Physical Review A: Atomic, Molecular and Optical Physics, vol. 72, no. 1, Article ID 012104, 2005.

[13] M. M. Stetsko and V. M. Tkachuk, "Perturbation hydrogenatom spectrum in deformed space with minimal length," Physical Review A: Atomic, Molecular and Optical Physics, vol. 74, no. 1, 2006.

[14] M. M. Stetsko, Physical Review A, vol. 74, Article ID 062105, 2006.

[15] F. Brau and F. Buisseret, "Minimal length uncertainty relation and gravitational quantum well," Physical Review D: Particles, Fields, Gravitation and Cosmology, vol. 74, no. 3, Article ID 036002, 2006.

[16] K. Nouicer, "Casimir effect in the presence of minimal lengths," Journal of Physics A: Mathematical and General, vol. 38, no. 46, pp. 10027-10035, 2005. 
[17] U. Harbach and S. Hossenfelder, "The Casimir effect in the presence of a minimal length," Physics Letters B, vol. 632, no. 2-3, pp. 379-383, 2006.

[18] S. Hossenfelder, "Interpretation of quantum field theories with a minimal length scale," Physical Review D: Particles, Fields, Gravitation and Cosmology, vol. 73, Article ID 105013, 2006.

[19] M. M. Stetsko and V. M. Tkachuk, "Scattering problem in deformed space with minimal length," Physical Review A: Atomic, Molecular and Optical Physics, vol. 76, no. 1, pp. 693695, 2007.

[20] S. Das, S. Ghosh, and D. Roychowdhury, "Relativistic thermodynamics with an invariant energy scale," Physical Review D: Particles, Fields, Gravitation and Cosmology, vol. 80, no. 12, 2009.

[21] D. Mania and M. Maziashvili, "Corrections to the black body radiation due to minimum-length deformed quantum mechanics," Physics Letters B, vol. 705, no. 5, pp. 521-528, 2011.

[22] B. R. Majhi and E. C. Vagenas, "Modified dispersion relation, photon's velocity, and unruh effect," Physics Letters B, vol. 725, no. 4-5, pp. 477-480, 2013.

[23] G. Yadav, B. Komal, and B. R. Majhi, "Rainbow Rindler metric and Unruh effect," International Journal of Modern Physics A, vol. 32, no. 33, 2017.

[24] S. Doplicher, K. Fredenhagen, and J. E. Roberts, "Spacetime quantization induced by classical gravity," Physics Letters B, vol. 331, no. 1-2, pp. 39-44, 1994.

[25] J. Magueijo and L. Smolin, "Generalized Lorentz invariance with an invariant energy scale," Physical Review D: Particles, Fields, Gravitation and Cosmology, vol. 67, no. 4, Article ID 044017, 2003

[26] G. Amelino-Camelia, J. Ellis, N. E. Mavromatos, D. V. Nanopoulos, and S. Sarkar, "Tests of quantum gravity from observations of big gamma-ray bursts," Nature, vol. 393, pp. 763-765, 1998.

[27] L. Barosi, F. A. Brito, and A. R. Queiroz, "Noncommutative field gas driven inflation," Journal of Cosmology and Astroparticle Physics, 2008.

[28] K. Nozari, M. A. Gorji, A. Damavandi Kamali, and B. Vakili, "Entropy bound for the photon gas in noncommutative spacetime," Astroparticle Physics, Elsevier, vol. 82, pp. 66-71, 2016.

[29] A. F. Ali and M. Moussa, "Towards thermodynamics with generalized uncertainty principle," Advances in High Energy Physics, vol. 2014, Article ID 629148, 7 pages, 2014.

[30] A. Camacho and A. Macías, "Thermodynamics of a photon gas and deformed dispersion relations," General Relativity and Gravitation, vol. 39, no. 8, pp. 1175-1183, 2007.

[31] G. Amelino-Camelia, International Journal of Modern Physics $D$, vol. 12, Article ID 1633, 2003.

[32] G. Amelino-Camelia, "Special treatment," Nature, vol. 418, no. 6893, pp. 34-35, 2002.

[33] G. Amelino-Camelia, "Relativity in space-times with shortdistance structure governed by an observer-independent (Planckian) length scale," International Journal of Modern Physics D, vol. 11, Article ID 0012051, pp. 35-60, 2002.

[34] J. Kowalski-Glikman and S. Nowak, "Non-commutative spacetime of Doubly Special Relativity theories," International Journal of Modern Physics D, vol. 12, pp. 299-316, 2003.

[35] K. A. Alam and M. M. Faruk, "On Boundedness of Entropy of Photon Gas in Noncommutative Spacetime," Advances in High Energy Physics, vol. 2017, Article ID 7289625, 2017.

[36] R. K. Pathria, Statistical Mechanics, Butterworth-Heinemann, Oxford, 1996.
[37] S. Das and R. K. Bhaduri, "Dark matter and dark energy from a Bose - Einstein condensate," Classical and Quantum Gravity, vol. 32, no. 10, Article ID 105003, 2015. 

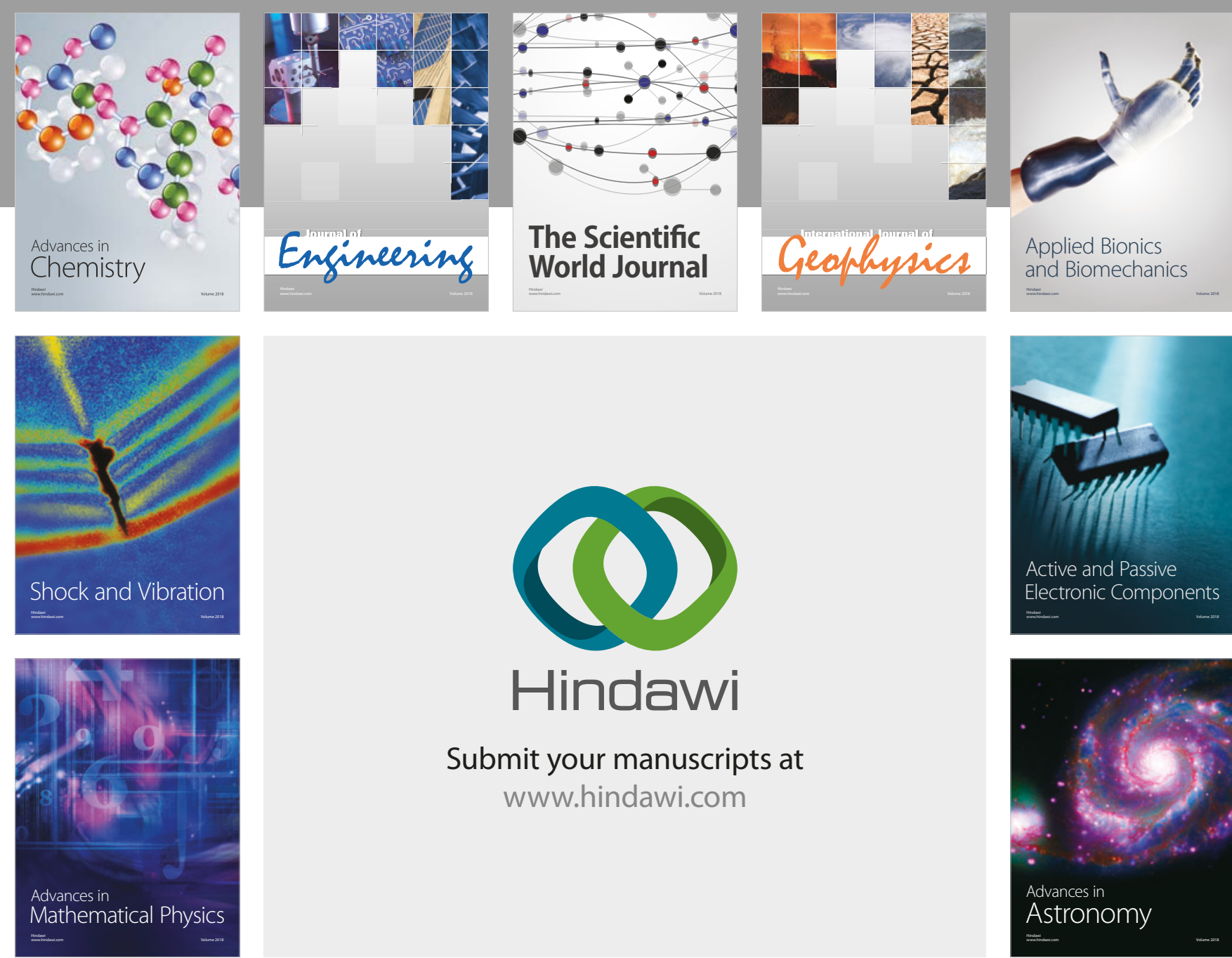

Submit your manuscripts at

www.hindawi.com

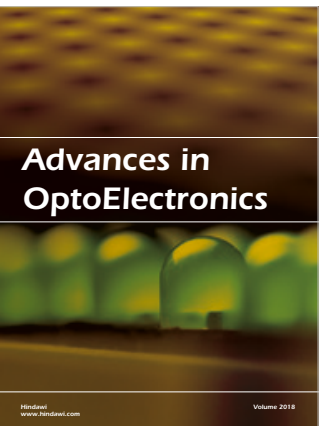

\section{Rotcting Machinery}
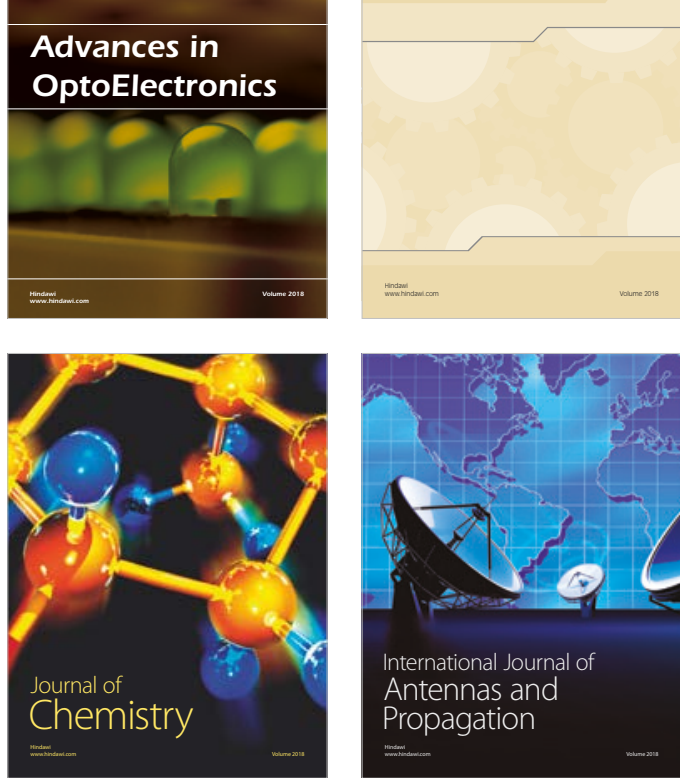

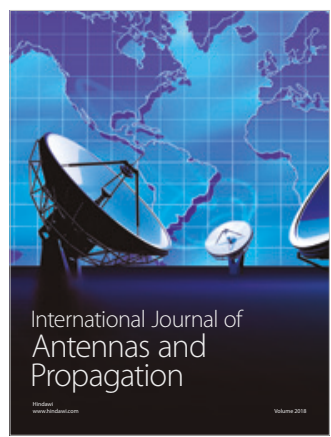

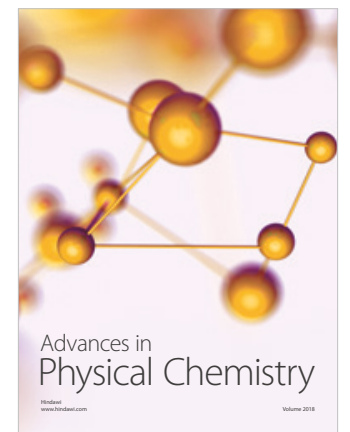

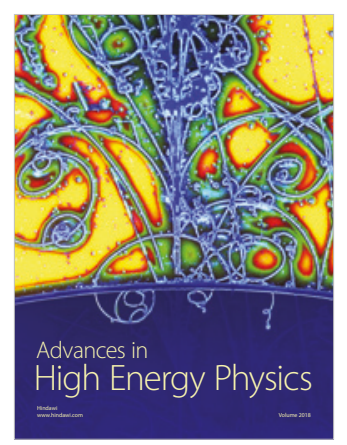

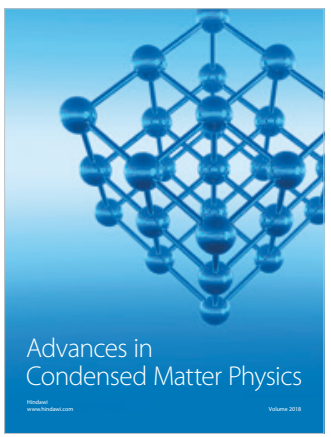

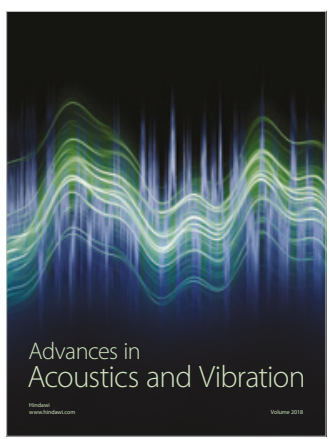

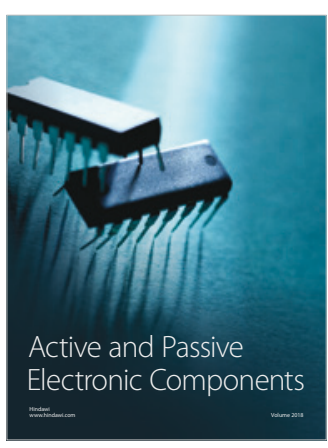
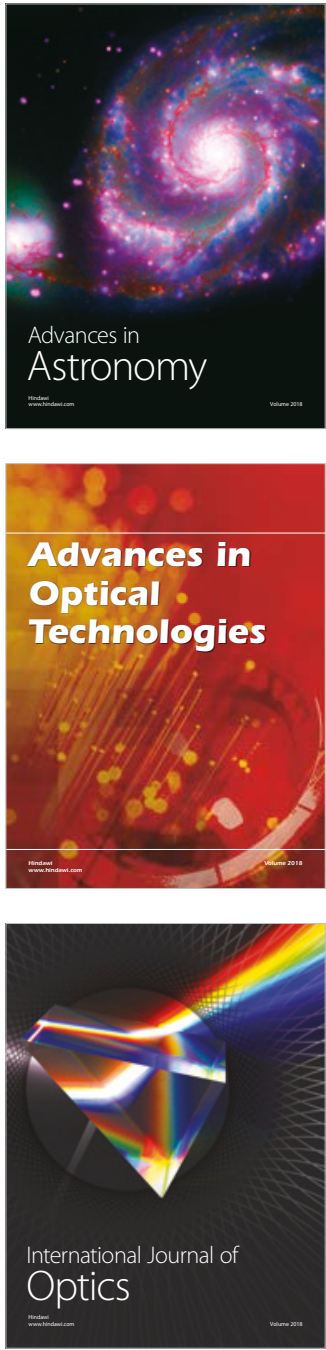